Article

\title{
Application of Genetic Algorithm Elements to Modelling of Rotation Processes in Motion Transmission Including a Long Shaft
}

\author{
Andriy Chaban ${ }^{1}$, Marek Lis ${ }^{2}$, Andrzej Szafraniec ${ }^{1, *(1)}$ and Radoslaw Jedynak ${ }^{1}$ \\ 1 Faculty of Transport, Electrical Engineering and Computer Science, Kazimierz Pulaski University of \\ Technology and Humanities, Malczewskiego 29, 26-600 Radom, Poland; atchaban@gmail.com (A.C.); \\ jedynak@uthrad.pl (R.J.) \\ 2 Faculty of Electrical Engineering, Czestochowa University of Technology, Al. Armii Krajowej 17, \\ 42-201 Czestochowa, Poland; lism@el.pcz.czest.pl \\ * Correspondence: a.szafraniec@uthrad.pl
}

check for updates

Citation: Chaban, A.; Lis, M.;

Szafraniec, A.; Jedynak, R.

Application of Genetic Algorithm Elements to Modelling of Rotation Processes in Motion Transmission Including a Long Shaft. Energies 2021, 14, 115. https://doi.org/10.3390/en 14010115

Received: 5 November 2020 Accepted: 23 December 2020 Published: 28 December 2020

Publisher's Note: MDPI stays neutral with regard to jurisdictional claims in published maps and institutional affiliations.

Copyright: (c) 2020 by the authors. Licensee MDPI, Basel, Switzerland. This article is an open access article distributed under the terms and conditions of the Creative Commons Attribution (CC BY) license (https: / / creativecommons.org/ licenses/by/4.0/).

\begin{abstract}
Genetic algorithms are used to parameter identification of the model of oscillatory processes in complicated motion transmission of electric drives containing long elastic shafts as systems of distributed mechanical parameters. Shaft equations are generated on the basis of a modified Hamilton-Ostrogradski principle, which serves as the foundation to analyse the lumped parameter system and distributed parameter system. They serve to compute basic functions of analytical mechanics of velocity continuum and rotational angles of shaft elements. It is demonstrated that the application of the distributed parameter method to multi-mass rotational systems, that contain long elastic elements and complicated control systems, is not always possible. The genetic algorithm is applied to determine the coefficients of approximation the system of Rotational Transmission with Elastic Shaft by equivalent differential equations. The fitness function is determined as leastsquare error. The obtained results confirm that application of the genetic algorithms allow one to replace the use of a complicated distributed parameter model of mechanical system by a considerably simpler model, and to eliminate sophisticated calculation procedures and identification of boundary conditions for wave motion equations of long elastic elements.
\end{abstract}

Keywords: mathematical modelling; genetic algorithm; long shaft equations; Hamilton-Ostrogradski principle; analytical mechanics; computer simulation

\section{Introduction}

Electric drives are among the top consumers of electricity, estimated to use $60 \%$ of the energy generated at present. A variety of drives are used in almost every industrial plant. A generalised electric drive consists of a drive motor, motion transmission system, loading system, and drive control system [1,2]. Each component has an impact on effective operations of the drive. Their adequate cooperation is crucial for optimal operation of the drive.

Drive motion transmission is emphasized in this paper. There are a number of motion transmission types in practice, depending on: motor power, type of motor loading system, system operating conditions, and the requirements and objectives of an electric drive [3-7]. Motion transmission in systems comprising long shafts is an important practical issue, since such systems are often used, e.g., in industrial conditions. These systems are used where loading mechanisms are far away from drive motors, most commonly in connection with production process requirements. This is particularly true of: high-power vertical pumps, turbine sets, lifts, rolling mills, diggers, etc. As motion transmission is the main factor of correct and efficient drive operation, dynamic processes across shafts must definitely be taken into account. 
Mathematical modelling of dynamic processes in long motion transmission shafts of electric drives is well discussed [4,8-10] using Lagrange's theory for lumped parameter systems and mechanics of physical continuum. In simple cases, shafts are divided into several absolutely rigid inertial units connected by means of elastic clutches. Each unit is described with second type Lagrange equations. In another, more complicated case, the shaft is treated as a single-dimensional (one spatial coordinate) continual system and described with fractional equations [4,8-11]. This approach poses the problem of computing boundary conditions, which is not always an easy task [4,11-13].

Another approach is applied to describe real physical processes in a drive system, since the long shaft equation might address wave processes in the shaft continuum. Application of Lagrange's theory to lumped parameter systems may be sufficient in a majority of cases, while results are satisfactory and agree with reality to a sufficient extent. The theory of continuum systems must be used in some precise drives, however. If, in line with Lagrange's theory, a shaft model is represented as a dual mass holonomic system (two degrees of freedom), velocity and rotation angle diagrams can be plotted for both the masses, in this case, the left and right shaft ends. If the shaft is presented as a continuum, on the other hand, and the results for velocity and rotation angle are compared to results obtained from an equivalent dual mass holonomic system, there will be significant discrepancies between the amplitudes of rotor and loading machine at the shaft ends, and the frequency of changes of these speeds. [14]. An angle $\delta$ is a measure of absolute error of the velocities. It will expand as time increases. The extreme of velocity or rotation angle function calculated by means of Lagrange's equations will in fact be shifted by $\delta$. This is very important in drive control. Supplying signal to a drive motor at a wrong instant shifted by $\delta$, for instance to damp resonance, may cause malfunction of the drive system. A somewhat different approach is proposed here to compute real velocities and rotational angles of shaft ends with the aid of the genetic algorithm.

\section{Mathematical Model of an Elastic-Dissipation Long Shaft of Distributed Parameters}

A mathematical model of the long shaft is based on an interdisciplinary method of modelling that involves modifications to the integral variational Hamilton-Ostrogradski principle by expanding the conservative Lagrange's function with two components. The first includes energy of dissipation forces, the other energies of external non-potential forces. This method has been introduced in [4]. The idea of modifying the Hamilton-Ostrogradski principle is similar to that applied in [8,9], where two additional components were introduced to the conservative Lagrangian formally, while the introduction gained mathematical foundations in [4]. Additionally, [8,9] analyse lumped parameter dynamic systems, and both lumped and distributed parameter dynamic systems.

Accordingly, the long shaft equation will be formulated as follows [4]:

$$
\frac{\partial^{2} \varphi}{\partial t^{2}}=a^{2} \frac{\partial^{2} \varphi}{\partial x^{2}}+\frac{\xi}{\rho J_{p}} \frac{\partial^{3} \varphi}{\partial x^{2} \partial t}-\frac{v_{e}}{\rho J_{p}} \frac{\partial \varphi}{\partial t}-\frac{1}{\rho J_{p}} m_{0}, a=\sqrt{G / \rho},
$$

where $a$-mechanical wave velocity; $\rho$-density of shaft material; $J_{p}$-polar shaft moment of inertia; $G$-shear modulus; $v_{e}, \zeta$ - external and internal shaft dispersion factors; $M_{0}$-load torque; $m_{0}$-load torque along each shaft point.

Direct boundary conditions for Euler-Poisson Equation (1), normally based on d'Alembert's theory $[4,8,9]$, are used here to develop a shaft model.

In general, a long shaft is analysed as the motion transmission part of an electric drive. The shaft connects the drive motor and the loading system (Figure 1). 


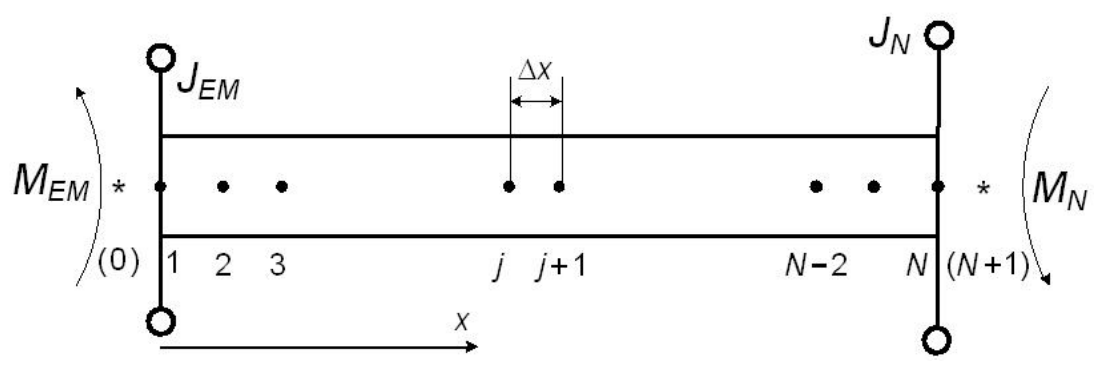

Figure 1. Long motion transmission shaft of electric drive.

The following nomenclature is introduced in Figure $1 M_{E M}$-electromagnetic torque of drive motor; $M_{N}$-drive loading torque; $J_{E M}, J_{N}$-moments of inertia of motor rotor and loading system; $x$-spatial coordinate; $N$-number of discrete nodes in (2); $\Delta x$-discretisation step of spatial derivatives; $\{(0)$ and $(N+1)\}$-fictitious discretisation nodes of spatial derivatives [4].

For the drive in Figure 1, (1) is simplified to:

$$
\frac{\partial^{2} \varphi}{\partial t^{2}}=a^{2} \frac{\partial^{2} \varphi}{\partial x^{2}}+\frac{\xi}{\rho J_{p}} \frac{\partial^{3} \varphi}{\partial x^{2} \partial t}
$$

Boundary conditions for (2) are found from equality of electromagnetic torque, elasticity and dissipation across elementary cross-sections of shaft ends and equality of loading torques, elasticity and dissipation.

$$
\begin{gathered}
\left.J_{E M} \frac{\partial^{2} \varphi}{\partial t^{2}}\right|_{x=0}-\left.G J_{p} \frac{\partial \varphi}{\partial x}\right|_{x=0}-\left.\xi \frac{\partial^{2} \varphi}{\partial x \partial t}\right|_{x=0}=M_{E M} \\
\left.J_{N} \frac{\partial^{2} \varphi}{\partial t^{2}}\right|_{x=l}+\left.G J_{p} \frac{\partial \varphi}{\partial x}\right|_{x=l}+\left.\xi \frac{\partial^{2} \varphi}{\partial x \partial t}\right|_{x=l}=M_{N}
\end{gathered}
$$

The system of Equations (2)-(4) is solved by discretising the spatial derivatives using the straight line method. This ultimately results in:

$$
\begin{gathered}
\frac{d \omega_{1}}{d t}=\frac{2\left(\Delta x M_{E M}-J_{p} G\left(\varphi_{1}-\varphi_{2}\right)-\xi\left(\omega_{1}-\omega_{2}\right)\right)}{\left(J_{p} \rho \Delta x+2 J_{E M}\right) \Delta x} \\
\frac{d \omega_{j}}{d t}=\frac{G}{\rho} \frac{\varphi_{j-1}-2 \varphi_{j}+\varphi_{j+1}}{(\Delta x)^{2}}+\frac{\xi}{\rho J_{p}} \frac{\omega_{j-1}-2 \omega_{j}+\omega_{j+1}}{(\Delta x)^{2}}, j=2,3 \ldots, N-1 \\
\frac{d \omega_{N}}{d t}=\frac{2\left(M_{N} \Delta x+J_{p} G\left(\varphi_{N-1}-\varphi_{N}\right)+\xi\left(\omega_{N-1}-\omega_{N}\right)\right)}{\left(J_{p} \rho \Delta x+2 J_{N}\right) \Delta x} \\
\frac{d \varphi_{j}}{d t}=\omega_{j}, \quad j=1,2, \ldots, N
\end{gathered}
$$

Velocity and rotation angle of shaft start (rotor) $-\omega_{1}, \varphi_{1}$ and their equivalents for the shaft end $-\omega_{N}, \varphi_{N}$ are the most interesting functions in (5)-(8). These are starting parameters for determining the instant of supplying the control signal in precise electric drives.

The logical question arises of a possible similarity between a long shaft in drive motion transmission and a dual mass elastic dissipation system [15-18]. It is not always the case, since similarity of a lumped and dispersed parameter system does not address velocity of mechanical wave motion, which leads to inaccuracies in determination of real system parameters. The angle $\delta$ appears among the calculation results, introducing a difference between velocities and rotation angles. 
A model of the system in Figure 1 is developed in its dual mass version.

$$
\begin{gathered}
\frac{d \omega_{1}}{d t}=\frac{1}{J_{E M}^{\prime}}\left(M_{E M}-c_{1,2}\left(\varphi_{1}-\gamma_{N}\right)-v_{1,2}\left(\omega_{1}-\omega_{N}\right)\right) \\
\frac{d \omega_{N}}{d t}=\frac{1}{J_{N}^{\prime}}\left(c_{1,2}\left(\varphi_{1}-\varphi_{N}\right)+v_{1,2}\left(\omega_{1}-\omega_{N}\right)-M_{N}\right) \\
\frac{d \varphi_{1}}{d t}=\omega_{1}, \quad \frac{d \varphi_{N}}{d t}=\omega_{N} \\
c_{1,2}=\frac{G J_{p}}{L}, v_{1,2}=\frac{\xi}{L}, \quad J_{E M}^{\prime}=J_{E M}+0,5 \rho J_{p} L \\
J_{N}^{\prime}=J_{N}+0,5 \rho J_{p} L, \quad J_{p}=\frac{\pi D^{4}}{32}
\end{gathered}
$$

where $c_{1,2}$-rigidity coefficient of equivalent elastic clutch; $v_{1,2}$-dispersion coefficient of equivalent elastic clutch; $L$-shaft length; $D$ —shaft diameter.

The mathematical model of electric drive motion transmission including long elastic elements is described with (2) and is the most accurate, which means results of computer simulation of functional dependences sought on its basis can be with great adequacy regarded as an experiment. The functions computed in this way will be approximated by Equation (18).

\section{Principles of Calculating Approximation Coefficients}

Initial attempts at solving the problem employed the classic approximation method using the least-squares method [19]. Since the proposed approximation function cannot be reduced by substitution to a linear or polynomial function with their ready-made equations, the definition was considered. This approximation problem can be approached as a non-linear least-squares method [19].

The approximation function is:

$$
f=f(x, \boldsymbol{\beta})
$$

where the vector $\beta$ stores $m$ of sought factors; $x$-function argument.

Parameter values need to be found for this function that 'best' match the data. Matching of a model and a data point is measured with its residual value, defined as the difference between the real dependent variable and its value anticipated by the model:

$$
r_{i}=y_{i}-f\left(x_{i}, \boldsymbol{\beta}\right), i=1, \ldots, n
$$

where $y_{i}-i^{\text {th }}$ value of the real function; $r_{i}-i^{\text {th }}$ difference (residue); $n$-number of measurement points.

The least-squares method finds optimum parameter values by minimising the total residues squared:

$$
S_{\mathrm{SUM}}=\sum_{i=1}^{n} r_{i}^{2} \rightarrow \min
$$

Extreme of function $S_{\mathrm{SUM}}$ can be found with the aid of the necessary condition, that is, by comparing its gradient to zero [19]. Since the model contains ' $m$ ' parameters $\boldsymbol{\beta}_{i}$, the same number of equations results.

The gradient of (16) will be computed considering (15)

$$
\frac{\partial S_{\mathrm{SUM}}}{\partial \beta_{i}}=2 \sum_{i=1}^{n} r_{i} \frac{\partial r_{i}}{\partial \beta_{j}}=2 \sum_{i=1}^{n} r_{i} \frac{\partial}{\partial \beta_{j}}\left(y_{i}-f\left(x_{i}, \boldsymbol{\beta}\right)\right)=-2 \sum_{i=1}^{n} r_{i} \frac{\partial f\left(x_{i}, \boldsymbol{\beta}\right)}{\partial \beta_{j}}=0, \quad j=1, \ldots, m
$$

Dependences of the approximated functions relating to the long motion transmission shaft are determined using the theory of a dual mass rotation system [14]. We additionally 
assume, in the general case, the moments of inertia may be different. Function $\omega(t)$ describes angular velocities of the left and right shaft ends.

$$
\omega(t)=C_{1} \exp \left(C_{2} t\right) \sin \left(C_{3} t\right)+C_{4} t
$$

where $C_{1}, \ldots, C_{4}$-approximation factors.

The approximation function is introduced as a general solution to a homogeneous (at $\mathrm{C} 4=0$ ) ordinary second-order equation with constant coefficients which describes oscillation processes in a dual mass system. If the right-hand side of the equation is constant, then $\mathrm{C} 4 \neq 0$.

Application of the non-linear least-squares method to find the parameters $\mathrm{C} 1, \mathrm{C} 2, \mathrm{C} 3$, C4, yields:

$$
\begin{gathered}
\frac{\partial S(\mathbf{C})}{\partial C_{1}}=-2 \sum_{i=1}^{n} \exp \left(C_{2} t_{i}\right) \sin \left(C_{3} t_{i}\right)\left[\omega_{i}-C_{1} \exp \left(C_{2} t_{i}\right) \sin \left(C_{3} t\right)-C_{4} t\right]=0 \\
\frac{\partial S(\mathbf{C})}{\partial C_{2}}=-2 C_{1} \sum_{i=1}^{n} t_{i} \exp \left(C_{2} t_{i}\right) \sin \left(C_{3} t_{i}\right)\left[\omega_{i}-C_{1} \exp \left(C_{2} t_{i}\right) \sin \left(C_{3} t\right)-C_{4} t\right]=0 \\
\frac{\partial S(\mathbf{C})}{\partial C_{3}}=-2 C_{1} \sum_{i=1}^{n} t_{i} \exp \left(C_{2} t_{i}\right) \cos \left(C_{3} t_{i}\right)\left[\omega_{i}-C_{1} \exp \left(C_{2} t_{i}\right) \sin \left(C_{3} t\right)-C_{4} t\right]=0 \\
\frac{\partial S(\mathbf{C})}{\partial C_{4}}=-2 \sum_{i=1}^{n} t_{i}\left[\omega_{i}-C_{1} \exp \left(C_{2} t_{i}\right) \sin \left(C_{3} t\right)-C_{4} t\right]=0 \\
\frac{\partial S_{\text {sum }}}{\partial \beta_{i}} \equiv F(\beta), \quad \beta \equiv \mathbf{C}=\left[C_{1}, C_{2}, C_{3}, C_{4}\right]^{\mathrm{T}}
\end{gathered}
$$

The iterative Gauss-Newton method serves to solve this system:

$$
\mathbf{C}^{k+1}=\mathbf{C}^{k}-\left[\mathbf{F}^{\prime}\left(\mathbf{C}^{k}\right)\right]^{-1} \mathbf{F}\left(\mathbf{C}^{k}\right)
$$

where $\mathbf{F}^{\prime}(\mathbf{C})$-Jacobi's matrix, $k$-number of iteration.

The procedure of numerically solving a similar set of non-linear equations in Mathematica software (Wolfram Research, Champaign, IL, USA) was presented in $[20,21]$.

In previous research it was proven that the system could not be solved numerically using the above method. The method often does not converge in practical calculations, since the fitness function (sum of squared residual values) is strongly non-linear, which is the case of our calculations. Thus in previous work we experienced problems with fitting the model to experimental. An improved method was then applied: Levenberg-Marquardt iterative algorithm that combines features of the greatest (gradient) decline and GaussNewton methods. It additionally uses the Hessian matrix of the system $(\mathrm{H})$, that is, information on its second fractional derivatives.

$$
\mathbf{C}^{k+1}=\mathbf{C}^{k}-\left[\mathbf{H}\left(\mathbf{C}^{k}\right)+\lambda \operatorname{diag} \mathbf{H}\left(\mathbf{C}^{k}\right)\right]^{-1} \nabla \mathbf{F}\left(\mathbf{C}^{k}\right),
$$

where $\mathbf{H}(\mathbf{C})$-Hessian matrix; $\nabla$-Hamilton operator; $\lambda$ - a certain positive constant.

Levenberg-Marquardt algorithm is a powerful calculation method that includes convergence of Newton's method on the one hand and methods of gradient descent on the other. The Levenberg-Marquardt method can be applied to solve equations with significant non-linearity.

Yet the Levenberg-Marquardt method is difficult to be implemented for this application. Therefore we have turned our attention to the alternative method of tackling the problem, namely we focused on the possibility to use Genetic algorithm (GA) for finding the values of model parameters. 


\section{Evolutionary Algorithm Approximating Angular Velocity of a Long Shaft}

The issue of determining parameters of an approximation function of shaft motion can be seen as a typical problem of global optimisation. We applied classical version of a genetic algorithm [20] to find parameters of a function describing the shaft's angular velocity. Genetic algorithm (GA) is a metaheuristic algorithm that helps to solve a range of complex problems, where a method of solving is not specified or known in advance, yet ways of evaluating quality of solutions are known.

In work [22], the usefulness of the genetic algorithms was for offline identification of electromagnetic and mechanical parameters of a mathematical model of induction machine, taking into account system dynamics (e.g., Coulomb's friction). Identification was performed using data acquired during a test consisting of a transient from standstill to a certain speed and successive free motion to standstill. The work [23] compares the performance of genetic algorithms and the Levenberg-Marquardt method for identification of parameters in the lumped model of an induction motor using transient stator current waveforms. The authors have found out that both methods are directly applicable to a variety of estimation problems found in the analysis of equivalent diagrams of electric machines. In work [24] it was used genetic algorithms for parameter identification for field control of induction motors. The authors have found out that the solutions of the problem depended heavily on the mathematical model and its measured data. They suggested to use the model equations in relative units in order to improve the accuracy and the efficiency of the identification method. The work [25] uses the real version of genetic algorithms implemented in the free Matlab toolkit (Mathworks Inc., Natick, MA, USA).

In the context of the subject of the present paper it is worth to notice that a somewhat similar identification problem in civil engineering (parameter estimation of internal thermal mass of building dynamic models for prediction of transient heating or cooling in a distributed network model of a building) has been tackled successfully in the paper by [26]. In work [27], the parameters of the hysteresis model for both soft and hard ferromagnets were estimated using the genetic algorithm method. The authors compared the performance of three meta-heuristic approaches (Genetic Algorithms and two more recent optimization techniques, namely Differential Evolution and Artificial Bee Colony).

On the basis of the afore-given literature review it can be stated that despite the genetic algorithms technique is a bit outdated and in some applications it has been successfully superseded with more recent optimization methods, it is still a useful tool for engineering purposes. A state-of-the-art paper, [28], comments on the advances in the field of natureinspired metaheuristics and we allow ourselves to refer the readers more interested in the subject to it for a detailed information on the advantages and the shortcomings of these methods.

Limiting possible values of all approximation parameters to a relatively narrow range is key to the optimisation of a problem. This means certain restrictions must be placed on values subject to genetic operators. These restrictions were initially determined on the basis of shaft motion simulation graphs.

This approach sees the process of optimisation as universal and nearly independent from a specific problem at hand. The objective function is designed in such a way as to meet typical requirements of solutions proposed. Finding a set of approximation function factors for which the total reference value deviations from values derived from approximation (absolute errors) squared is minimum is a universally acceptable criterion of the approximation problem solution. In our case, data from the shaft simulation computed on their basis of (5)-(8) are the point of reference. In earlier studies the objective function referred to the maximum relative error of approximation.

The objective function in the case of $C_{4}=0$, see (18), becomes:

$$
f_{\text {celu }}\left(C_{1}, C_{2}, C_{3}\right)=\sum_{i=1}^{n}\left[\omega_{i}-C_{1} \exp \left(C_{2} t_{i}\right) \sin \left(C_{3} t_{i}\right)\right]^{2} \rightarrow \min
$$


and in the case $C_{4} \neq 0$

$$
f_{\text {celu }}\left(C_{1}, \ldots, C_{4}\right)=\sum_{i=1}^{n}\left[\omega_{i}-C_{1} \exp \left(C_{2} t_{i}\right) \sin \left(C_{3} t_{i}\right)-C_{4} t_{i}\right]^{2} \rightarrow \min
$$

A general diagram of the genetic algorithm employed in this paper is illustrated in Figure 2 .

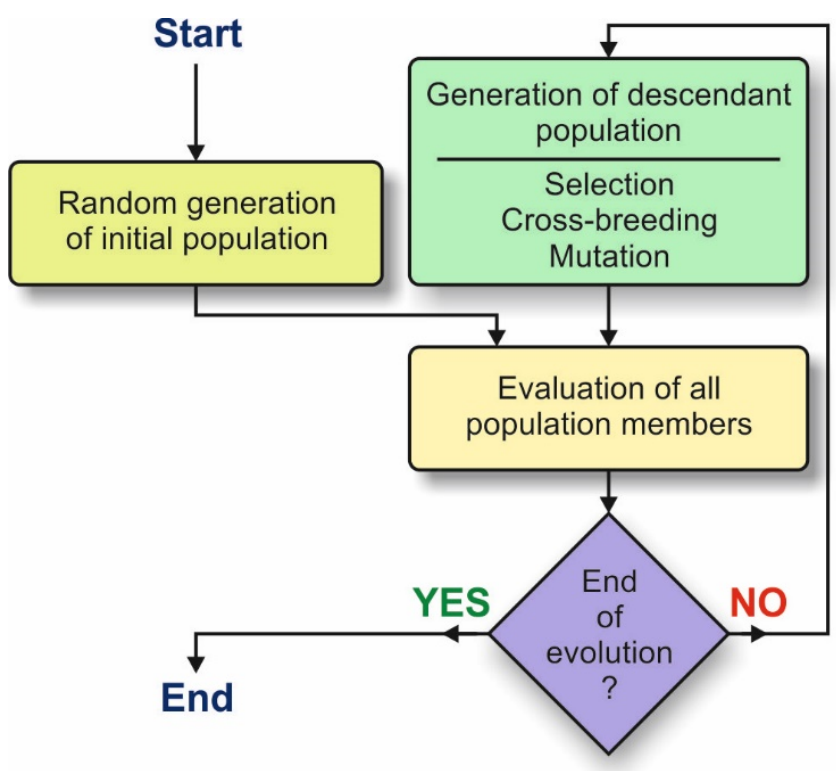

Figure 2. GA flow diagram.

In our simulations we have availed of freely available GAOT (Mathworks Inc., Natick, MA, USA) toolbox, which can be applied with practically any version of Matlab (more recent versions of Matlab offer a Global Optimization toolbox, which contains implementations of genetic algorithms and pattern search). We have found that the GAOT toolbox is simple to use and sufficient for our needs.

Evaluation of all population members is based on selection of a function objective minimisation. A range of simulations were undertaken before finally selecting values of the different parameters included in Table 1 and used in our approach based on the genetic algorithm.

Table 1. Genetic algorithm parameters.

\begin{tabular}{cc}
\hline Parameter & Value \\
\hline Lower parameter limits: $\left(\mathrm{C}_{1}, \mathrm{C}_{2}, \mathrm{C}_{3}, \mathrm{C}_{4}\right)$ & $(-1,-3,100,0)$ \\
Upper parameter limits: $\left(\mathrm{C}_{1}, \mathrm{C}_{2}, \mathrm{C}_{3}, \mathrm{C}_{4}\right)$ & $(1,0,120,1)$ \\
Number of generations & 1000 \\
Size of population & 60 \\
Method of selection & Roulette \\
Probability of cross-breeding & 0.8 \\
Probability of mutation & 0.2 \\
Accuracy & $10^{-6}$ \\
\hline
\end{tabular}

The upper and lower boundaries were determined on the basis of simulation testing. $\mathrm{C} 1$ depends on function amplitude, $\mathrm{C} 2$ on dissipation coefficient (where dissipation is absent, $\mathrm{C} 2=0$ ), $\mathrm{C} 3$ depends on frequency of process oscillation (i.e., coefficient of shaft rigidity). 
Root mean square error $(\Delta)$ is calculated:

$$
\Delta=\sqrt{\frac{\sum_{i=1}^{n}\left(\omega_{i}-\omega_{a i}\right)^{2}}{n(n-1)}}
$$

where $\omega_{a}$-function approximated by means of GA.

Rotation angles for the shaft ends are important dependences. They are derived from: $\omega(t)=\frac{d \varphi(t)}{d t}[4]:$

$$
\varphi(t)=\left.\int_{0}^{t} \omega(t)\right|_{t=\tau} d \tau=\left.C_{1} \int_{0}^{t} \exp \left(C_{2} t\right) \sin \left(C_{3} t\right)\right|_{t=\tau} d \tau+\left.C_{4} \int_{0}^{t} t\right|_{t=\tau} d \tau
$$

Integration of (18) by parts and the necessary transformations produce an expression serving to compute the function of shaft rotation angle:

$$
\varphi(t)=\frac{C_{1}}{C_{2}^{2}+C_{3}^{2}}\left[C_{3}+\exp \left(C_{2} t\right)\left(C_{2} \sin \left(C_{3} t\right)-C_{3} \cos \left(C_{3} t\right)\right)\right]+\frac{C_{4}}{2} t^{2}
$$

\section{Results of Computer Simulation}

The electromechanical system was presented as a motion transmission including a long shaft. The torque generated by the electric motor was applied to the left end and the loading system to the right end. To simplify the calculations, the state with a constant starting torque and loading is taken into consideration. Three experiments were undertaken with the above torques applied to the shaft ends (c.f. Figure 1). In the first experiment, the torques are: $M_{E M}=M_{N}=1000 \mathrm{~N} \cdot \mathrm{m}$, and in the second $M_{E M}=1000 \mathrm{~N} \cdot \mathrm{m}$ $M_{N}=1004 \mathrm{~N} \cdot \mathrm{m}$. If moments of inertia are equal, coefficients of approximation will be equal in terms of their absolute values (after the modulus), but with different signs. This is the general approach that is used in the paper for different moments of inertia. The drive will exhibit zero velocity and a constant shaft torsion angle in a steady state as part of experiment one, whereas it will accelerate in the steady state as part of the second experiment. When the third experiment is in progress, given the same torques as in the second experiment, the rotation angle is determined on the basis of the dual mass model (see Equations (9)-(13)) and the genetic algorithm. The parameters of the long shaft and the reference unit are shown in Table 2.

Table 2. Model parameters.

\begin{tabular}{ccc}
\hline Parameter & Symbol & Value \\
\hline Number of discretisation nodes & $N$ & 90 \\
Shear modulus & $G$ & $8.1 \times 1010 \mathrm{~N} \cdot \mathrm{m}$ \\
Density of shaft material & $\rho$ & $7850 \mathrm{~kg} / \mathrm{m}^{3}$ \\
Shaft diameter & $D$ & $0.05 \mathrm{~m}$ \\
Shaft length & $L$ & $4.5 \mathrm{~m}$ \\
Shaft internal scattering factor & $\xi$ & $0.5 \mathrm{~N} \cdot \mathrm{m}^{2}$ \\
Discretisation step of spatial derivatives & $\Delta x$ & $0.05 \mathrm{~m}$ \\
Moment of rotor inertia & $J_{E M}$ & $20 \mathrm{~N} \cdot \mathrm{m}^{2}$ \\
Moment of loading system inertia & $J_{N}$ & $20 \mathrm{~N} \cdot \mathrm{m}^{2}$ \\
\hline
\end{tabular}

\section{Results of Computer Simulation for the Dual Mass Model}

The system of differential Equations (5)-(8) was solved by means of 4th order RungeKutta method including the time step $\Delta t=5 \cdot 10^{-7} \mathrm{~s}$. The system (9)-(11) was also solved by means of 4th order Runge-Kutta method, though with a different time step $\Delta t=5 \times 10^{-5} \mathrm{~s}$. This shift of the time step helped to avoid latent integration methods [4]. 
Approximated functions of velocity and rotation angle were derived from (18), (30). The constants $\mathrm{C} 1, \mathrm{C} 2, \mathrm{C} 3$, and $\mathrm{C} 4$ are:

For the first experiment-left shaft end:

$$
C=(+0.46137829,-1.34580552,+108.1684494,0) \text {; }
$$

For the first experiment-right shaft end:

$$
C=(-0.461378372,-1.34580652,+108.168452,0) \text {; }
$$

For the second experiment-left shaft end:

$$
C=(+0.46230207,-1.34582264,+108.168436,+0.0994407489) ;
$$

For the second experiment-right shaft end:

$$
C=(-0.46229961,-1.34577768,+108.168432,+0.0994406377) \text {. }
$$

Figure 3 depicts instant angular velocity of the left shaft end (towards the motor) during the first experiment, determined according to the shaft Equations (5)-(8) and the mathematical model of motion transmission (18). This dependence is virtually identical in both the cases of modelling. For the right shaft end (towards the load), the speed function is practically the same, though in counter-phase to the left end's velocity. The graph shows the system enters a steady state after $5 \mathrm{~s}$. Since both the torque moments are identical in experiment one, the shaft is not turning.

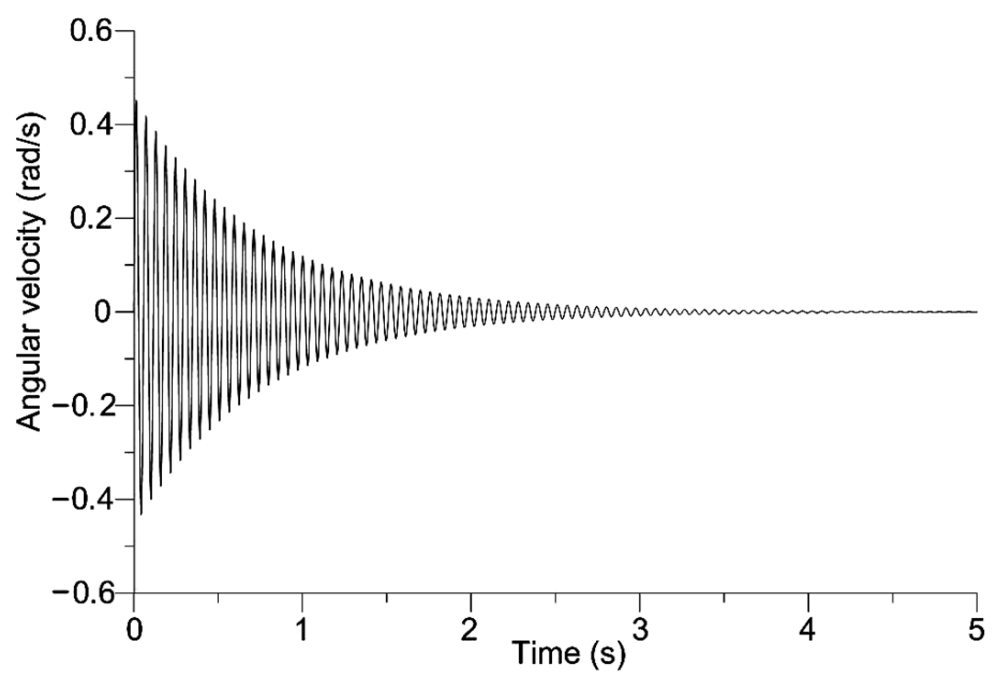

Figure 3. Instant angular velocity of the motion transmission left end for the first experiment.

Figures 4 and 5 present instant rotation angles of both the shaft ends in the first experiment, derived from (5)-(8) and (29). Comparative analysis of the graphs demonstrates operating principles of the transmission. First, oscillation rotation angles are similar and both the functions are in counter-phase to each other. Second, the left and right shaft ends in the steady state can be seen to be turned by an angle of approximately 0.004 rad relative to the zero point of the system of coordinates. This means the total angle of shaft torsion in relation to its ends is approximately $0.008 \mathrm{rad}$. 


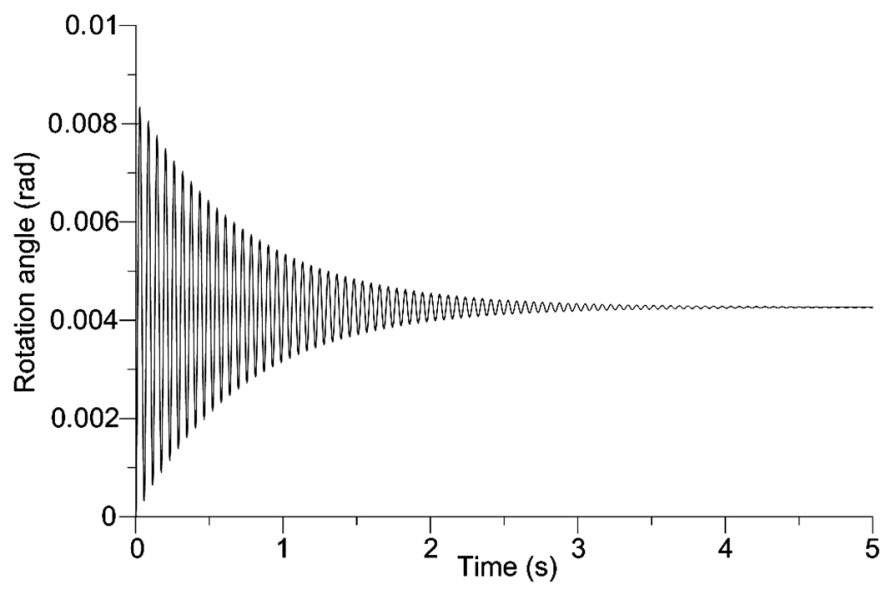

Figure 4. Instant rotation angle of the motion transmission left end in the first experiment.

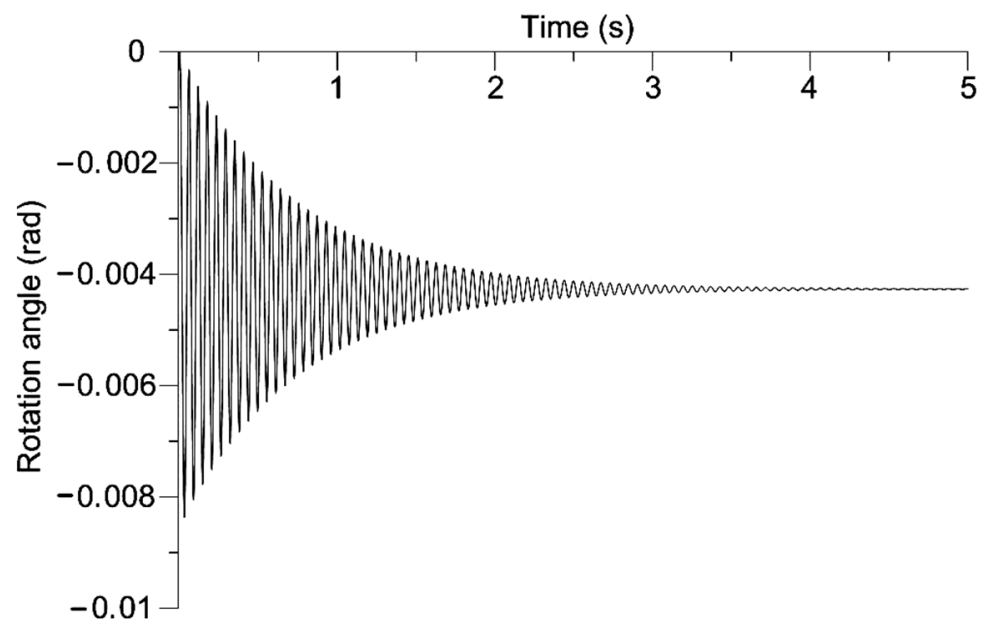

Figure 5. Instant rotation angle of the motion transmission right end in the first experiment.

Figure 6 illustrates instant angular velocity of the motion transmission left end for the second experiment. As both the torsion torques are different, the shaft continues to accelerate. The rotation angle derived from the genetic algorithm is virtually the same as the speed determined by means of fundamental equations of the analytical continuum mechanics.

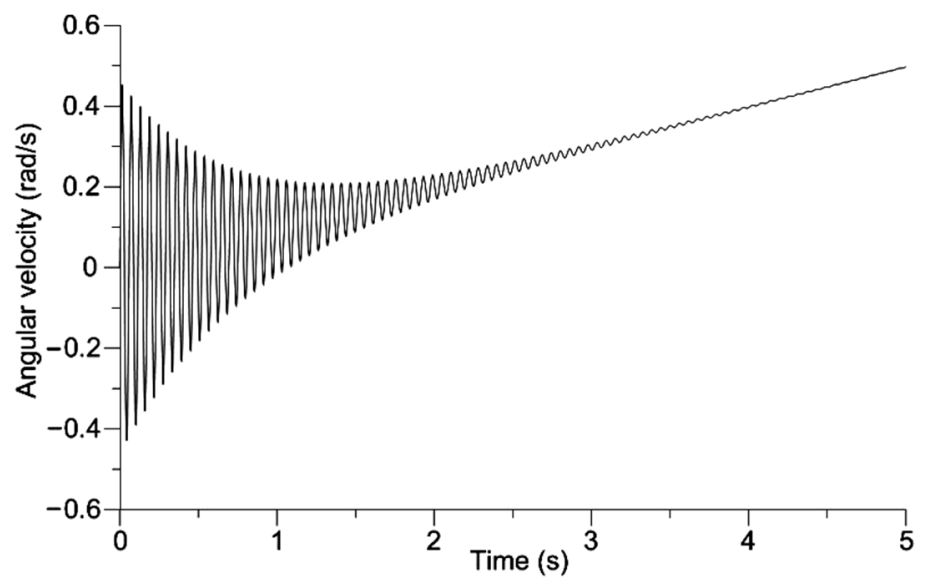

Figure 6. Instant angular velocity of the motion transmission left end for the second experiment. 
Instant rotation angles of both the shaft in the time interval $t \in(0 ; 1) \mathrm{s}$ are presented in Figures 7 and 8. A comparison detects major differences. Both the functions not only are in counter-phase but also display other amplitudes in the time range under discussion.

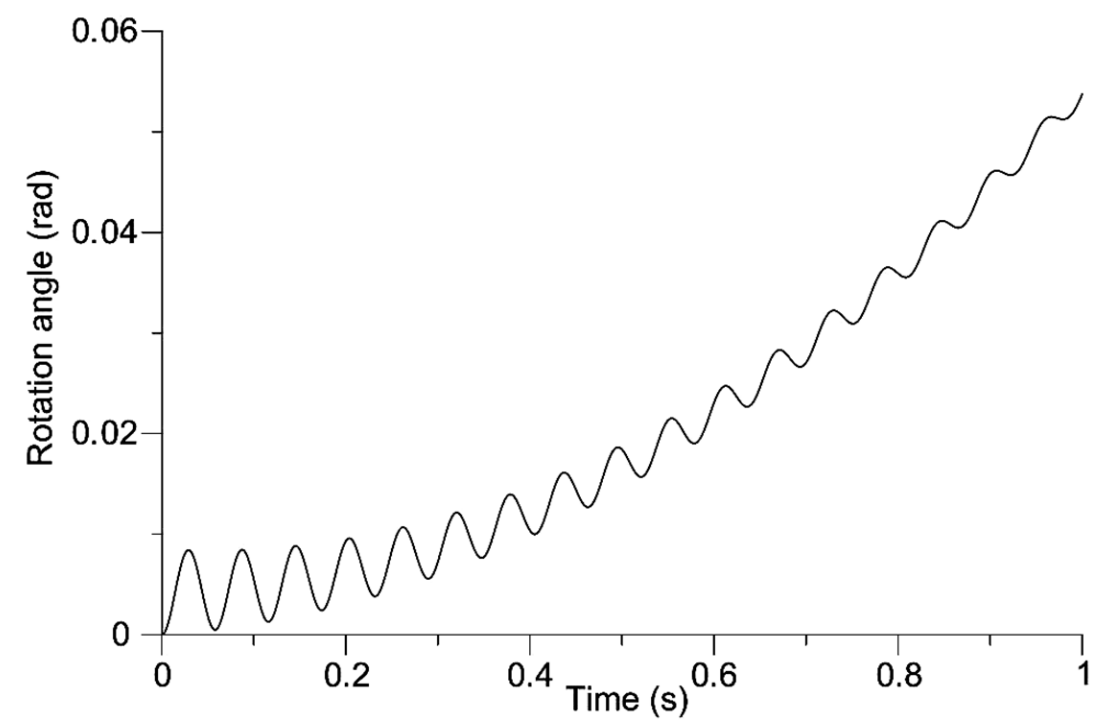

Figure 7. Instant rotation angle of the motion transmission left end in the second experiment $t \in(0 ; 1) \mathrm{s}$.

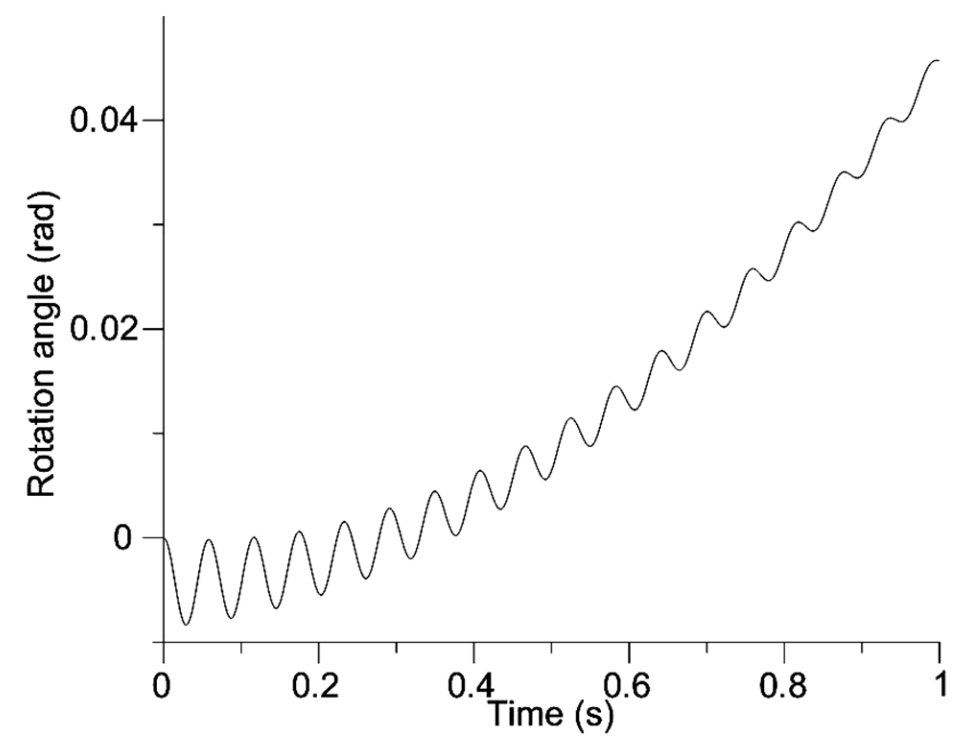

Figure 8. Instant rotation angle of the motion transmission right end in the second experiment $t \in(0 ; 1) \mathrm{s}$.

Figures 9 and 10 show rotation angles of both the shaft ends in the time range $t \in(0 ; 1)$ $\mathrm{s}$ of the third experiment. The following nomenclature can be noted: $\varphi_{1}(t)$-rotation angle based on the dual mass system model, Equations (9)-(13), and $\varphi_{2}(t)$-rotation angle based on the genetic algorithm model (30). 


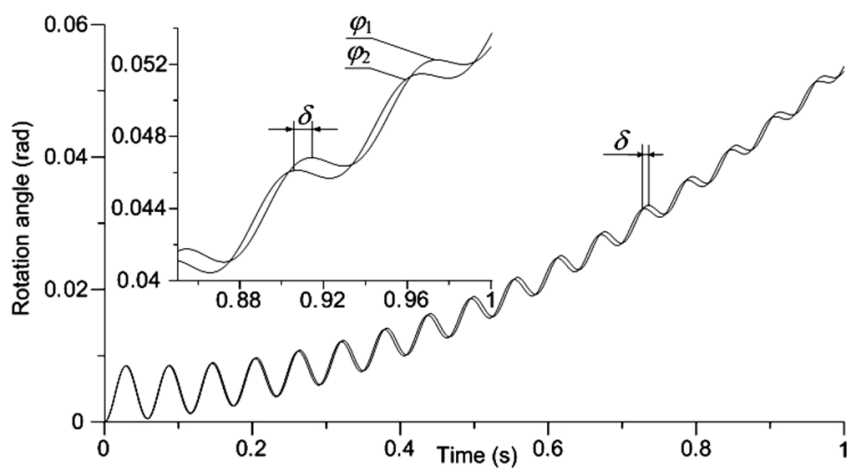

Figure 9. Instant rotation angle of the motion transmission left end in the third experiment $t \in(0 ; 1) \mathrm{s}$, $t \in(0.85 ; 1) \mathrm{s}$.

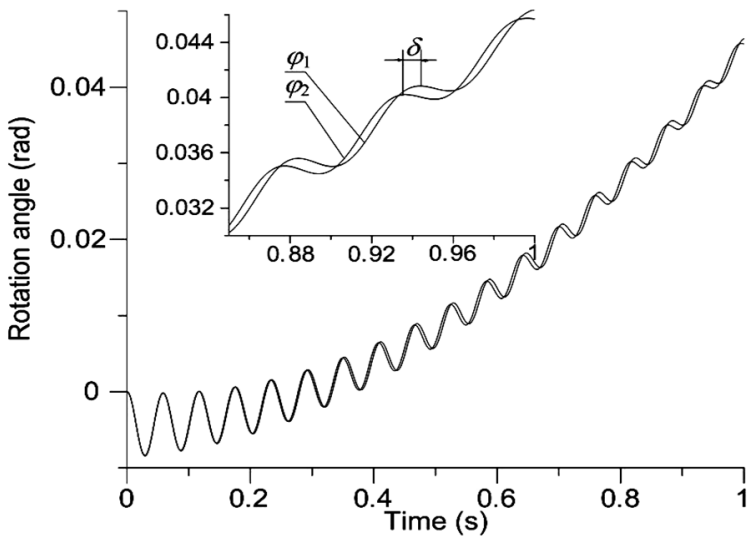

Figure 10. Instant rotation angle of the motion transmission right end in the third experiment $t \in(0 ; 1) \mathrm{s}, t \in(0.85 ; 1) \mathrm{s}$.

For the sake of improved visualisation of physical processes in the drive motion transmission in Figures 9 and 10, the same dependences are illustrated in the time interval $t \in(0.85 ; 1) \mathrm{s}$.

The time delay angle $\delta$ can be seen in both the graphs. The physical interpretation of this process is well known. A mechanical wave moving along a shaft produces the delay. Meanwhile, the transmission model based on a dual mass system assumes no such notion as velocity of wave motion. It is evident the rotation angle function based on the dual mass model does not correspond to the real value of $\delta$. This causes supply of control signal to the drive motor to be inaccurate or occasionally incorrect in time. This is one of the main reasons why other approaches to simplification of the motion transmission model are employed as part of distributed parameter systems. Application of the genetic algorithm has broad prospects in distributed parameter systems.

Results of relative error analysis of approximating formulas for the first and second experiments at given angular velocities of both the shaft ends are included in Table 3. Duration of the experiments was $t \in(0 ; 5) \mathrm{s}$. The number of approximation points: $n=1000$. The table provides simulation results for the time interval $t \in(0 ; 25) \mathrm{s}$. Relative errors $\varepsilon$ are computed as follows:

$$
\varepsilon=\frac{\omega_{a i}-\omega_{i}}{\omega_{i}}
$$


Table 3. Relative errors $\varepsilon$ for the first and second experiments at given angular velocities of both the shaft ends in the time interval $\Delta t=0.25 \mathrm{~s}$

\begin{tabular}{ccccc}
\hline \multirow{2}{*}{$(\mathbf{s})$} & \multicolumn{2}{c}{ First Experiment } & \multicolumn{2}{c}{ Second Experiment } \\
\cline { 2 - 5 } & Left End & Right End & Left End & Right End \\
\hline 0 & 0 & 0 & 0 & 0 \\
0.25 & -0.0000034 & -0.0000037 & 0.00000417 & 0.000000225 \\
0.5 & -0.000043 & -0.000041 & -0.000086 & -0.000032 \\
0.75 & 0.000186 & 0.000182 & -0.00035 & -0.000012 \\
1 & -0.0000017 & -0.0000019 & -0.00001 & 0.000125 \\
1.25 & 0.004583 & 0.004609 & -0.000024 & 0.0000224 \\
1.5 & -0.000083 & -0.000086 & 0.00000401 & -0.0000002 \\
1.75 & -0.00068 & -0.00068 & 0.00000113 & -0.000015 \\
2 & 0.00152 & 0.001507 & -0.000011 & 0.00000638 \\
2.25 & 0.001601 & 0.001599 & 0.00000079 & 0.00000305 \\
2.5 & -0.00087 & -0.00084 & 0.00000113 & -0.0000016 \\
2.75 & -0.00051 & -0.00052 & -0.0000026 & -0.000001 \\
3 & 0.000417 & 0.00042 & 0.00000233 & 0.00000104 \\
3.25 & -0.00066 & -0.00069 & 0.00000229 & -0.00000052 \\
3.5 & 0.000408 & 0.000405 & -0.00000036 & -0.00000069 \\
3.75 & 0.002241 & 0.002262 & 0.00000083 & 0.000000313 \\
4 & 0.002611 & 0.002598 & 0.00000171 & -0.00000014 \\
4.25 & -0.00153 & -0.00153 & 0.000000693 & -0.00000042 \\
4.5 & -0.00017 & -0.00023 & 0.00000071 & -0.000000011 \\
4.75 & -0.00042 & -0.00043 & 0.00000125 & -0.00000006 \\
5 & -0.00075 & -0.00073 & 0.000001 & -0.00000024 \\
\hline & & & & \\
\hline
\end{tabular}

Table 3 indicates values of the angular velocity are virtually identical. Root mean square error for the approximating formulas in both the experiments was also calculated using (28). The results are part of Table 4.

Table 4. Root mean square errors for the first and second experiments at given angular velocities of both the shaft ends in the time interval $\Delta t=0.005 \mathrm{~s}$.

\begin{tabular}{cccc}
\hline \multicolumn{2}{c}{ First Experiment } & \multicolumn{2}{c}{ Second Experiment } \\
\hline Left End & Right End & Left End & Right End \\
\hline 0.000159 & 0.000172 & $4.74741 \times 10^{-6}$ & $6.07118 \times 10^{-6}$ \\
\hline
\end{tabular}

\section{Experimental Testing and Verification of Calculation Results}

The experimentation was conducted on a laboratory stand for testing of drive systems including long elastic elements [29]. A flow diagram of the stand is shown in Figure 11.

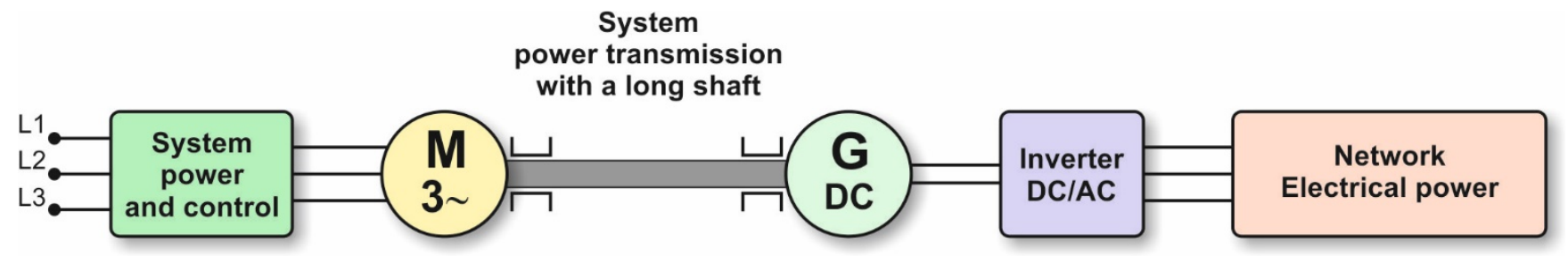

Figure 11. Flow diagram of a laboratory stand for testing of drive systems including elastic connections.

The drive system in testing consists of a three-phase induction motor connected to loading with an elastic element. The moment of motion transmission system inertia is modified by mounting additional steel discs on the shaft towards the loading system. The laboratory stand is equipped with two pulse angle position encoders (incremental 
encoders) with 3600 pulses per rotation. The moment is measured with a torque meter affixed to the motor shaft. Signals from measurement sensors are transmitted to a computer having two National Instruments PCle-6351 measurement cards. LabVIEW (National Instruments, Austin, TX, USA) software is employed to gather data.

These are the long shaft's parameters: $l=0.66 \mathrm{~m}, D=0.025 \mathrm{~m}, \rho=7850 \mathrm{~kg} / \mathrm{m}^{3}$, $J=0.0001987 \mathrm{kgm}^{2}, G=8.11010 \mathrm{~N} / \mathrm{m}^{2}$. Default parameters of the motor supplying power to the drive system: $P_{N}=7.5 \mathrm{~kW}, U_{N}=400 \mathrm{~V}, I_{N}=17.5 \mathrm{~A}, n_{N}=14.661 / \mathrm{s}, J_{1}=0.0952 \mathrm{kgm}^{2}$. Default parameters of the direct current generator loading the system: $P_{N}=12 \mathrm{~kW}$, $n_{N}=22.511 / \mathrm{s}, U_{N}=220 \mathrm{~V}, I_{N}=64 \mathrm{~A}, U_{f}=220 \mathrm{~V}, I_{f}=1.21 \mathrm{~A}, J_{G}=0.11 \mathrm{kgm}^{2}$.

Numerical calculations were carried out for a shaft length $4.5 \mathrm{~m}$ and diameter $0.05 \mathrm{~m}$. Experimentation on a shaft of such large dimensions involves a great risk of torsional moments that may cause a breakdown. Therefore, a long elastic shaft of a length $0.66 \mathrm{~m}$ and thickness $0.025 \mathrm{~m}$ was used in the testing.

Laboratory results are presented graphically and compared to the numerical calculations. Examples of laboratory test results compared to the corresponding numerical results are illustrated in Figure 12, Figure 13, Figure 14, Figure 15. The drive system was loaded with a default moment $M_{L}=81 \mathrm{~N} \cdot \mathrm{m}$.

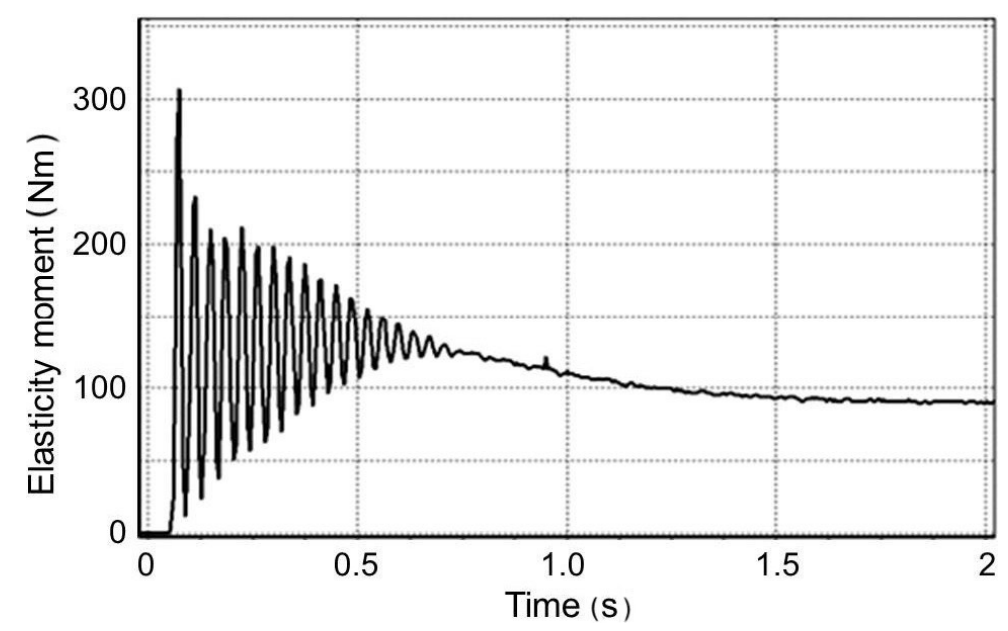

Figure 12. Measurement results of elasticity moment in a long-shaft drive transmission system.

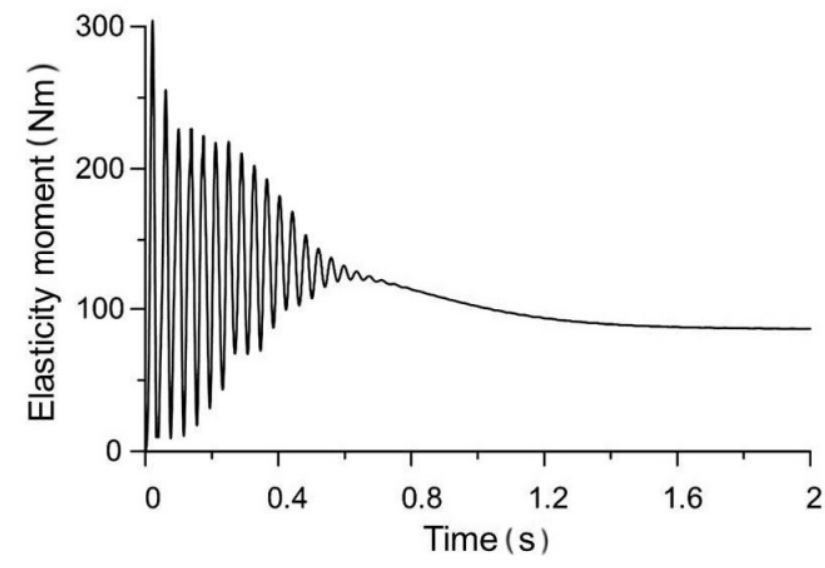

Figure 13. Results of numerical simulation of elasticity moment in a long-shaft drive transmission system. 


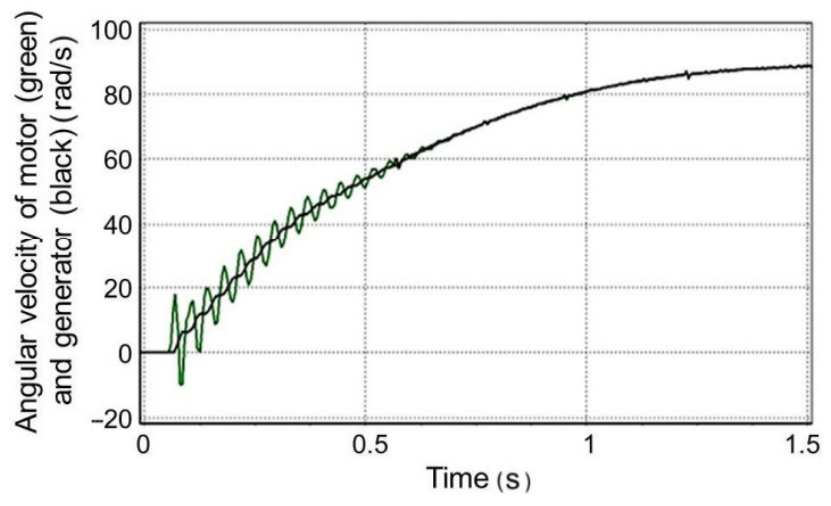

Figure 14. Measurement results of angular velocity of motor $\omega_{1}$ and generator loading the drive system $\omega_{2}$.

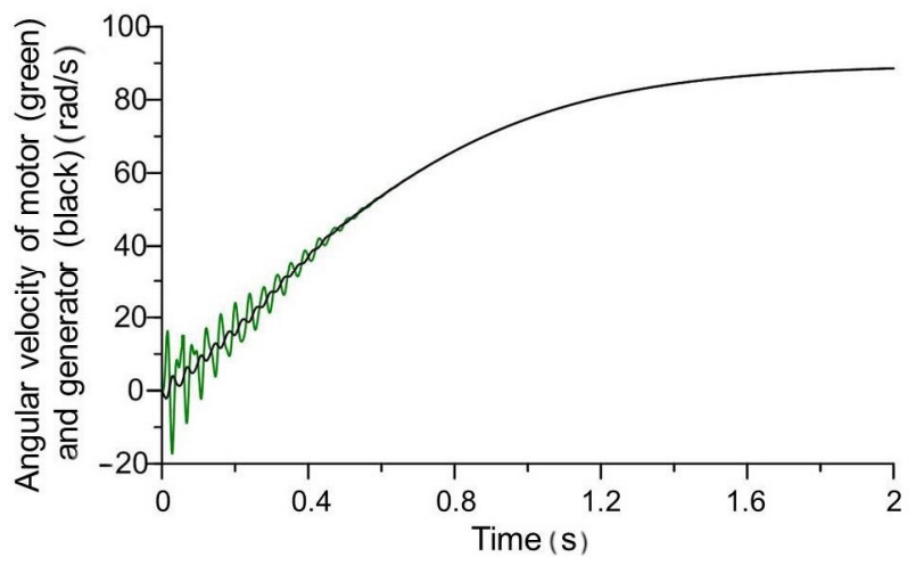

Figure 15. Results of numerical simulation of angular velocity of motor $\omega_{1}$ and generator loading the drive system $\omega_{2}$.

A simulation for these measurement cases was undertaken using the authors' mathematical models in order to determine degree of matching between the calculation and measurement results. The verification concerned instant values of elasticity moment across the element connecting the motor and loading and the motor's angular velocity.

In order to compare calculation results and measurement results recorded on the test stand, mean values of the first five amplitudes of elasticity moment and angular velocity oscillations were determined. The difference between numerical calculation results and measurement results was 3.1\% for elasticity moment and $19.1 \%$ for angular velocity, evidence of a high adequacy of our mathematical models based on variational approaches. Such accuracy is sufficient for the purposes of designing new and modernisation of existing drive systems.

\section{Conclusions}

Elements of genetic algorithm can be used with success with the aid of parametric identification of approximation coefficients for functions of rotational speed and rotation angles of ends of motion transmission across long elastic elements. This avoids the complicated theory of oscillation process modelling in long shafts at the level of the distributed parameter system.

Experience shows replacement of long elastic elements with distributed mechanical parameters with multiple mass elastic-dissipative units of lumped parameters, for instance elastic clutches, is not always right. This is connected with the fact a multiple (e.g., dual) mass interpretation of an equivalent model fails to include the important phenomenon of mechanical wave motion along a shaft, which will delay the oscillation process in a clutch 
being modelled. This physical phenomenon is at the root of the theory of infinite degrees of freedom in lumped parameter systems; contrary to multiple mass models where number of degrees of freedom is pre-determined and equal to number of generalised coordinates for holonomic systems.

The computer simulation results give reasons to conclude the genetic algorithm is correctly used in this paper to identify function parameters of rotation speed and angle of shaft ends in the drive's motion transmission. The error of approximation is to the order of $10^{-4}$ for the first experiment and $10^{-6}$ for the second, which confirms the long shaft model developed by the authors can be used in precise operating conditions of electric drives containing long elastic elements of distributed parameters.

Author Contributions: Conceptualization, A.C., R.J., M.L. and A.S.; methodology, A.C. and M.L.; software, A.C. and R.J.; formal analysis, A.C., R.J. and A.S.; investigation, A.C., R.J., M.L. and A.S.; resources, A.C., R.J., M.L. and A.S.; data curation, A.C., R.J., M.L. and A.S.; writing-original draft preparation, A.C. and A.S.; writing-review and editing, A.C. and A.S.; visualization, A.C., M.L. and A.S.; supervision, A.C. and M.L.; project administration, A.C. and M.L.; funding acquisition, M.L. and A.S. All authors have read and agreed to the published version of the manuscript.

Funding: This research received no external funding.

Institutional Review Board Statement: Not applicable.

Informed Consent Statement: Not applicable.

Data Availability Statement: Data is contained within the article.

Conflicts of Interest: The authors declare no conflict of interest.

\section{References}

1. Lei, G.; Zhu, J.; Guo, Y.; Liu, C.; Ma, B. A Review of Design Optimization Methods for Electrical Machines. Energies 2017, 10, 1962. [CrossRef]

2. Li, X.; Chau, K.T.; Wang, Y. Modeling of a Field-Modulated Permanent-Magnet Machine. Energies 2016, 9, 1078. [CrossRef]

3. Chen, W.; Liang, J.; Shi, T. Speed Synchronous Control of Multiple Permanent Magnet Synchronous Motors Based on an Improved Cross-Coupling Structure. Energies 2018, 11, 282. [CrossRef]

4. Czaban, A. Hamilton-Ostrogradski Principle in Electromechanical Systems; T. Soroki: Lviv, Ukraine, $2015 ;$ p. 488.

5. Jesenik, M.; Hamler, A.; Trbušić, M.; Trlep, M. The Use of Evolutionary Methods for the Determination of a DC Motor and Drive Parameters Based on the Current and Angular Speed Response. Mathematics 2020, 8, 1269. [CrossRef]

6. Kanaan, H.Y.; Al-Haddad, K.; Roy, G. Analysis of the electromechanical vibrations in induction motor drives due to the imperfections of the mechanical transmission system. Math. Comput. Simul. 2003, 63, 421-433. [CrossRef]

7. Luo, W.; Wang, B.; Zhao, H.; Luo, Y. Modeling and simulation of non-linear dynamic process of the induction motor system with fluctuating potential loads. Sci. China Technol. Sci. 2014, 57, 1729-1737. [CrossRef]

8. White, D.C.; Woodson, H.H. Electromagnetic Energy Conversion; Wiley: New York, NY, USA, 1958.

9. Ortega, R.; Loria, A.; Nicklasson, P.J.; Sira-Ramirez, H. Passivity-Beast Control of Euler-Lagrange Systems: Mechanical, Electrical and Electromechanical Applications; Springer: London, UK, 1998; p. 543.

10. Goldberg, D.E.; Holland, J.H. Genetic Algorithms and Machine Learning. Mach. Learn. 1988, 3, 95-99. [CrossRef]

11. Wang, Y.; Liu, H.; Liu, H.; Zhao, J. Dynamic analysis and system identification of a synchronous belt mechanism driven by PMSM. In Proceedings of the 3rd IEEE International Conference on Control Science and Systems Engineering (ICCSSE), Beijing, China, 17-19 August 2017; pp. 364-368.

12. Dong, X.; Wang, C.; Yang, Q.; Si, W. System identification of distributed parameter system with recurrent trajectory via deterministic learning and interpolation. Nonlinear Dyn. 2018, 95, 73-86. [CrossRef]

13. Talaei, B.; Xu, H.; Jagannathan, S. Near optimal boundary control of distributed parameter systems modeled as parabolic pdes by using finite difference neural network approximation. In Proceedings of the 53rd IEEE Conference on Decision and Control, Los Angeles, CA, USA, 15-17 December 2014; pp. 6776-6781.

14. Lukasik, Z.; Chaban, A.; Szafraniec, A. Mathematical model of long-shaft electric drive transmission including concentrated and distributed parameter methods. Bull. Pol. Acad. Sci. Tech. Sci. 2020, in press.

15. Ito, K.; Watanabe, K.; Iwasaki, M. Robust load states estimation against mechanical parameters variation of a two-mass system using acceleration-aided dynamic Kalman filter. In Proceedings of the 15th International Workshop on Advanced Motion Control (AMC), Tokyo, Japan, 9-11 March 2018; pp. 491-496.

16. Orlowska-Kowalska, T.; Szabat, K. Neural-Network Application for Mechanical Variables Estimation of a Two-Mass Drive System. IEEE Ind. Electron. Mag. 2007, 54, 1352-1364. [CrossRef] 
17. Saarakkala, S.E.; Hinkkanen, M. Identification of Two-Mass Mechanical Systems Using Torque Excitation: Design and Experimental Evaluation. IEEE Trans. Ind. Appl. 2015, 51, 4180-4189. [CrossRef]

18. Popenda, A.; Lis, M.; Nowak, M.; Blecharz, K. Mathematical modeling of transient states in a drive system with a long elastic element. Energies 2020, 13, 1181. [CrossRef]

19. Kincaid, D.; Kincaid, D.R.; Cheney, E.W. Numerical Analysis-Mathematics of Scientific Computing. Math. Comput. 1992, 59, 297. [CrossRef]

20. Man, K.F.; Tang, K.S.; Kwong, S. Genetic Algorithms Concepts and Designs; Springer: London, UK, 2001; p. 346.

21. Walasek, J.; Jedynak, R. Inverse Langevin-like function for statistical description of the polymer chain in orienting fields. Int. J. Solids Struct. 2019, 163, 15-24. [CrossRef]

22. Alonge, F.; Raimondi, F.; Ferrante, G.; D'Ippolito, F. Parameter identification of induction motor model using genetic algorithms. IEE Proc. Control. Theory Appl. 1998, 145, 587-593. [CrossRef]

23. Shaw, S.R.; Leeb, S.B. Identification of induction motor parameters from transient stator current measurements. IEEE Trans. Ind. Electron. 1999, 46, 139-149. [CrossRef]

24. Huang, K.; Wu, Q.; Turner, D. Effective identification of induction motor parameters based on fewer measurements. IEEE Trans. Energy Convers. 2002, 17, 55-60. [CrossRef]

25. Chwastek, K.; Szczyglowski, J. Identification of a hysteresis model parameters with genetic algorithms. Math. Comput. Simul. 2006, 71, 206-211. [CrossRef]

26. Wang, S.; Xu, X. Parameter estimation of internal thermal mass of building dynamic models using genetic algorithm. Energy Convers. Manag. 2006, 47, 1927-1941. [CrossRef]

27. Jesenik, M.; Mernik, M.; Trlep, M. Determination of a Hysteresis Model Parameters with the Use of Different Evolutionary Methods for an Innovative Hysteresis Model. Mathematics 2020, 8, 201. [CrossRef]

28. Stork, J.; Eiben, A.E.; Bartz-Beielstein, T. A new taxonomy of global optimization algorithms. Nat. Comput. 2020, 1-24. [CrossRef]

29. Popenda, A. Mathematical modelling of transmission shafts based on electrical and mechanical similarities. Przeglad Elektrotechniczny 2019, 1, 198-201. [CrossRef] 